\title{
MORE ON CONVERGENCE IN UNITARY MATRIX SPACES
}

\author{
JONATHAN ARAZY ${ }^{1}$ \\ Abstract. Let $E$ be a symmetric sequence space satisfying the Radon-Riesz \\ Property$$
\left\{\left\|x_{n}\right\| \rightarrow\|x\| \text { and } x_{n} \rightarrow x \text { weakly }\right\} \Rightarrow\left\|x_{n}-x\right\| \rightarrow 0,
$$ \\ then the same is true for the associated unitary matrix space $C_{E}$.
}

Let $E$ be a symmetric sequence space, i.e., a Banach space of sequences so that the standard unit vectors $\left\{e_{n}\right\}_{n=1}^{\infty}$ (defined by $e_{n}(j)=\delta_{n, j}$ ) form a 1-symmetric, normalized basis of $E$. The unitary matrix space $C_{E}$ associated with $E$ is the Banach space of all compact operators $x$ on $l_{2}$ for which $s(x) \in E$, normed by

$$
\|x\|_{C_{E}}=\|s(x)\|_{E} \text {. }
$$

Here $s(x)=\left(s_{n}(x)\right)$ is the sequence of $s$-numbers of $x$, i.e., the eigenvalues of $\left(x^{*} x\right)^{1 / 2}$, arranged in a nonincreasing ordering, counting multiplicity.

In the recent paper [1] we reduce the study of certain properties of $C_{E}$ to the study of the analogous properties of $E$. One consequence is the following result which characterizes convergence in $C_{E}$ in terms of convergence in $E$ (see [2]): If $\left\{x_{n}\right\}$ and $x$ are elements of $C_{E}$, then $\left\|x_{n}-x\right\|_{C_{E}} \rightarrow 0$ if and only if $\left\|s\left(x_{n}\right)-s(x)\right\|_{E}$ $\rightarrow 0$ and $x_{n} \rightarrow x$ weakly.

In this note we apply [1] to study some related convergence property, and show that it extends from $E$ to $C_{E}$.

Definition. $A$ Banach space $X$ is said to have the Radon-Riesz Property (RRP, in short) if $\left\|x_{n}\right\| \rightarrow\|x\|$ and $x_{n} \rightarrow x$ weakly imply $\left\|x_{n}-x\right\| \rightarrow 0$ for all $\left\{x_{n}\right\}$ and $x$ in $X$.

THEOREM I. Let $E$ be a symmetric sequence space. Then $E$ has the RRP if and only if $C_{E}$ has the $R R P$.

This theorem extends the known result for the case $E=l_{p}, 1<p<\infty$, (see [3]; or apply the uniform convexity of the spaces $\left.C_{p}=C_{b},[4]\right)$, and answers affirmatively a question of $\mathrm{B}$. Simon.

If $C_{E}$ has the RRP, then the same is true for $E$ which is isometric to the subspace of $C_{E}$ consisting of all diagonal matrices. So the point in the theorem is that the RRP extends from $E$ to $C_{E}$.

Received by the editors June 4, 1980 and, in revised form, July 18, 1980.

AMS (MOS) subject classifications (1970). Primary 47D15; Secondary 46B99, 47B10.

Key words and phrases. Unitary matrix spaces, symmetric sequence spaces, compact operators on Hilbert space, Radon-Riesz Property.

${ }^{1}$ Research supported in part by NSF Grant No. MSC-7901771.

(c) 1981 American Mathematical Society 0002-9939/81/0000-0410/\$02.25 
Let us start with two propositions.

Proposition 1. Assume that $E$ satisfies the RRP. Let $x \in E,\|x\|=1$ and let $y \in E^{*}$ be a supporting functional for $x:\|y\|=1=\langle x, y\rangle$. Then $y$ does not vanish on the support of $x$, i.e., $x(i) \neq 0$ implies $y(i) \neq 0$.

Proof. The assertion is clear if $E=l_{1}$. If $E \neq l_{1}$ then $e_{n} \rightarrow 0$ weakly. If $x\left(i_{0}\right) \neq 0$ but $y\left(i_{0}\right)=0$, we get a contradiction as follows. Let $u=\sum_{i=1 ; i \neq i_{0}}^{\infty} x(i) e_{i}$. Then,

$$
1=\|x\| \geqslant\|u\| \geqslant|\langle u, y\rangle|=|\langle x, y\rangle|=1 \text {. }
$$

So, $\|u\|=1$. Define

$$
x_{n}=\sum_{\substack{i=1 \\ i \neq i_{0}}}^{n} x(i) e_{i}+x\left(i_{0}\right) e_{n+1} .
$$

Clearly, $\left\|x_{n}\right\| \rightarrow\|x\|=1=\|u\|$. Also $\sum_{i=1 ; i \neq i_{0}}^{n} x(i) e_{i} \rightarrow u$ in norm. So $x_{n} \rightarrow u$ weakly. But $\left\|x_{n}-u\right\| \rightarrow\left|x\left(i_{0}\right)\right|>0$, contradicting the RRP.

Proposition 2. Assume that $E$ has the RRP. Let $x \in E$ has nonnegative coordinates and let $\lambda>0$. Then for all $i$,

$$
\left\|x+\lambda e_{i}\right\|>\|x\| \text {. }
$$

Proof. Again, this is clear for $E=l_{1}$. Assume therefore that $E \neq l_{1}$. Clearly, $\left\|x+\lambda e_{i}\right\| \geqslant\|x\|$ for all $i$. If for some $i_{0}\left\|x+\lambda e_{i_{0}}\right\|=\|x\|$, we get a contradiction as follows. If $x\left(i_{0}\right)>0$, let $y \in E^{*}$ be a normalized element which supports $x:\langle x$, $y\rangle=\|x\|$. Clearly, we can assume that the coordinates of $y$ are nonnegative. By Proposition $1, y\left(i_{0}\right)>0$. So,

$$
\|x\|=\left\|x+\lambda e_{i_{0}}\right\| \geqslant\left|\left\langle x+\lambda e_{i_{0}}, y\right\rangle\right|=\|x\|+\lambda y\left(i_{0}\right)>\|x\|,
$$

a contradiction. If $x\left(i_{0}\right)=0$, define

$$
x_{n}=\sum_{i=1}^{n} x(i) e_{i}+\lambda e_{n+1} .
$$

Then $\left\|x_{n}\right\|=\left\|\sum_{i=1}^{n} x(i) e_{i}+\lambda e_{i_{0}}\right\| \rightarrow\left\|x+\lambda e_{i_{0}}\right\|=\|x\|$, and $x_{n} \rightarrow x$ weakly. But $\left\|x_{n}-x\right\| \rightarrow \lambda>0$, contradicting the RRP.

Proof of Theorem I. Let $\left\{x_{n}\right\}$ and $x$ be elements of $C_{E}$ satisfying

$$
\left\|x_{n}\right\| \rightarrow\|x\|, \quad x_{n} \rightarrow x \text { weakly. }
$$

If $\left\|x_{n}-x\right\| \nrightarrow 0$, then for some $\varepsilon>0$ and some subsequence $y_{k}=x_{n_{k}}-x$ we have $\left\|y_{k}\right\|>\varepsilon$ for all $k$.

Choose a matrix representation in which $x$ is diagonal (with the $s$-numbers on the diagonal). Since $y_{k} \rightarrow 0$ weakly, we can assume (by passing to a subsequence if necessary and by a standard perturbation argument) that $\left\{y_{k}\right\}$ is a shell-block sequence, i.e., that for some increasing sequence $\left\{m_{k}\right\}$ we have $y_{k}(i, j)=0$ if $\max \{i, j\} \leqslant m_{k}$ or $\max \{i, j\}>m_{m+1}$. By Theorem 2.4 of [1] we can assume, furthermore, that there exists a tensor product representation and a further subsequence, still denoted by $\left\{y_{k}\right\}$ for simplicity, so that

$$
y_{k}=\sum_{i=1}^{k-1}\left(a_{i} \otimes e_{k, i}+b_{i} \otimes e_{i, k}\right)+c_{k} \otimes e_{k, k}
$$


and

$$
x=\sum_{k=1}^{\infty} z_{k} \otimes e_{k, k}
$$

with $z_{k}$ diagonal matrices.

ClAMM. $a_{i}=b_{i}=0$ for all $i$.

Indeed, if $a_{i_{0}}(\nu, \mu)=\alpha \neq 0$ for some $i_{0}, \nu$ and $\mu$, we define $\tilde{z}_{i_{0}}$ by

$$
\tilde{z}_{i_{0}}(\mu, \mu)=\left(\left|z_{i_{0}}(\mu, \mu)\right|^{2}+|\alpha|^{2}\right)^{1 / 2}
$$

and

$$
\tilde{z}_{i_{0}}(s, t)=z_{i_{0}}(s, t) \text { otherwise. }
$$

Let

$$
\tilde{x}=\sum_{\substack{i=1 \\ i \neq i_{0}}}^{\infty} z_{i} \otimes e_{i, i}+\tilde{z}_{i_{0}} \otimes e_{i_{0}, i_{0}}
$$

By Proposition 2, $\|x\|<\|\tilde{x}\|$. On the other hand, if we define for $i_{0}<m$ a projection $P_{m}$ by

$$
P_{m}\left(\sum_{k, l} u_{k, l} \otimes e_{k, l}\right)=\sum_{k=1}^{m} u_{k, k} \otimes e_{k, k}+u_{m+1, i_{0}}(\nu, \mu) e_{\nu, \mu} \otimes e_{m+1, i_{0}}
$$

then $\boldsymbol{P}_{\boldsymbol{m}}$ is contractive and thus,

$$
\begin{aligned}
\left\|x_{n_{m}}\right\| & >\left\|P_{m} x_{n_{m}}\right\|=\left\|\sum_{k=1}^{m} z_{k} \otimes e_{k, k}+\alpha e_{\nu, \mu} \otimes e_{m+1, i_{0}}\right\| \\
& =\left\|\sum_{\substack{k=1 \\
k \neq i_{0}}}^{m} z_{k} \otimes e_{k, k}+\tilde{z}_{i_{0}} \otimes e_{i_{0}, i_{0}}\right\| \rightarrow\|\tilde{x}\| .
\end{aligned}
$$

This is a contradiction, since $\left\|x_{n_{m}}\right\| \rightarrow\|x\|<\|\tilde{x}\|$. This shows that $\alpha=0$. Thus $a_{i}=0$ for all $i$. The proof that $b_{i}=0$ for all $i$ is the same. This concludes the proof of the claim.

It follows that $y_{k}=c_{k} \otimes e_{k, k}$ for all $k$. Let $d_{k}$ be a diagonal matrix whose diagonal entries are the $s$-numbers of $c_{k}$, and define for $m=1,2, \ldots$

$$
u_{m}=\sum_{k=1}^{m-1} z_{k} \otimes e_{k, k}+d_{m} \otimes e_{m, m} \text {. }
$$

$u_{m}$ is a diagonal matrix, and thus we regard it as an element of $E$. Now,

$$
\begin{aligned}
\left\|\sum_{k=1}^{m-1} z_{k} \otimes e_{k, k}\right\| & \leqslant\left\|u_{m}\right\|=\left\|\sum_{k=1}^{m-1} z_{k} \otimes e_{k, k}+y_{m}\right\| \\
& =\left\|x_{n_{m}}-\sum_{k=m}^{\infty} z_{k} \otimes e_{k, k}\right\| .
\end{aligned}
$$

So,

$$
\left\|u_{m}\right\| \rightarrow\|x\| \text {. }
$$


Also, let $\beta=\sum_{k=1}^{\infty} \beta_{k} \otimes e_{k, k}$ be any diagonal matrix in $\left(C_{E}\right)^{*}=C_{E^{*}}$ (i.e., a member of $\left.E^{*}\right)$. For all $k$ let $v_{k}, w_{k}$ be unitary matrices so that $v_{k} c_{k} w_{k}=d_{k}$. Then

$$
\begin{aligned}
\left\langle d_{k} \otimes e_{k, k}, \beta\right\rangle & =\left\langle d_{k}, \beta_{k}\right\rangle=\operatorname{trace}\left(v_{k} c_{k} w_{k} \beta_{k}^{*}\right) \\
& =\operatorname{trace}\left(c_{k}\left(v_{k}^{*} \beta_{k} w_{k}^{*}\right)^{*}\right)=\left\langle c_{k}, v_{k}^{*} d_{k} w_{k}^{*}\right\rangle .
\end{aligned}
$$

Let $v=\sum_{k=1}^{\infty} v_{k} \otimes e_{k, k}, w=\sum_{k=1}^{\infty} w_{k} \otimes e_{k, k}$. Then $v, w$ are unitary an

$$
\left\langle d_{k} \otimes e_{k, k}, \beta\right\rangle=\left\langle y_{k}, v^{*} \beta w^{*}\right\rangle \rightarrow 0 \text {. }
$$

So, clearly,

$$
u_{m} \rightarrow x \text { weakly. }
$$

But

$$
\begin{aligned}
\left\|u_{m}-x\right\| & \geqslant\left\|d_{m} \otimes e_{m, m}\right\|-\left\|\sum_{k=m}^{\infty} z_{k} \otimes e_{k, k}\right\| \\
& \geqslant\left\|y_{m}\right\|-\varepsilon / 2>\varepsilon-\varepsilon / 2=\varepsilon / 2
\end{aligned}
$$

if $m$ is large enough. This, together with (*) and (**) contradicts the assumption that $E$ has the RRP, and thus concludes the proof of Theorem I.

In exactly the same way one can prove the following result.

THEOREM II. Let E be a symmetric sequence space. The following two statements are equivalent.

(1) If $\left\{x_{n}\right\}$ and $x$ are elements of $E$ satisfying

(†) $\left\|x_{n}\right\|_{E} \rightarrow\|x\|_{E}$ and $x_{n}(i) \rightarrow x(i)$ for all $i$, then $\left\|x_{n}-x\right\|_{E} \rightarrow 0$.

(2) If $\left\{x_{n}\right\}$ and $x$ are elements of $C_{E}$ satisfying

$(\dagger \dagger)\left\|x_{n}\right\|_{C_{E}} \rightarrow\|x\|_{C_{E}}$ and $x_{n} \rightarrow x$ in the weak operator topology, then $\left\|x_{n}-x\right\|_{C_{E}}$ $\rightarrow 0$.

Appendix. We decide to add to the paper a direct proof of Theorems I and II in the important special case of the trace class $C_{1}=C_{l_{1}}$, which does not depend on the elaborate blocking technique of [1]. The proof presented here is an improvement, due to B. Simon, of our original proof. We would like to thank Professor Simon for his comments and interest.

It is easy to see that (1) of Theorem II is satisfied in case $E=l_{1}$. So Theorems I and II in the case $E=l_{1}$ are reduced to the following

THEOREM. Let $\left\{x_{n}\right\}_{n=1}^{\infty} \subseteq C_{1}$ and $x \in C_{1}$ be so that

(i) $\left\|x_{n}\right\|_{1} \rightarrow\|x\|_{1}$;

(ii) $x_{n} \rightarrow x$ in the weak operator topology. Then $\left\|x_{n}-x\right\|_{1} \rightarrow 0$.

Here, $\|\cdot\|_{1}$ is, of course, the trace-norm, i.e.,

$$
\|x\|_{1}=\operatorname{trace}\left(x^{*} x\right)^{1 / 2}=\sum_{j=1}^{\infty} s_{j}(x) .
$$

The proof of the theorem is based on the following inequality, which is of independent interest. 
Proposition. Let $x \in C_{1}$, let $p$ be an orthogonal projection in $l_{2}$ and let $q=I-$ p. Then

(iii) $\|x\|_{1}^{2} \geqslant\|p x p\|_{1}^{2}+\|p x q\|_{1}^{2}+\|q x p\|_{1}^{2}+\|q x q\|_{1}^{2}$.

SKETCH OF PROOF. For every $y \in B\left(l_{2}\right)$ we have $\left(\|\cdot\|_{\infty}\right.$ is the operator norm)

$$
\begin{aligned}
\|y\|_{\infty} & =\sup \{\|y \xi\| ;\|\xi\| \leqslant 1\}=\sup \left\{\left(\|p y \xi\|^{2}+\|q y \xi\|^{2}\right)^{1 / 2} ;\|\xi\|<1\right\} \\
& \leqslant\left(\|p y\|_{\infty}^{2}+\|q y\|_{\infty}^{2}\right)^{1 / 2} .
\end{aligned}
$$

From this one gets

$$
\|y\|_{\infty} \leqslant\left(\|p y p\|_{\infty}^{2}+\|p y q\|_{\infty}^{2}+\|q y p\|_{\infty}^{2}+\|q y q\|_{\infty}^{2}\right)^{1 / 2}
$$

and from this (iii) follows by standard duality arguments.

PROOF OF THE THEOREM. By (ii), there exists an increasing sequence $\left\{p_{n}\right\}_{n=1}^{\infty}$ of finite rank orthogonal projections in $l_{2}$ so that $p_{n} \uparrow I$ and

(iv) $\left\|p_{n} x_{n} p_{n}-x\right\|_{1} \rightarrow 0$.

Let $q_{n}=I-p_{n}$. Then, by (iii)

$$
\begin{aligned}
\left\|x_{n}\right\|_{1}^{2} & \geqslant\left\|p_{n} x_{n} p_{n}\right\|_{1}^{2}+\left\|p_{n} x_{n} q_{n}\right\|_{1}^{2}+\left\|q_{n} x_{n} p_{n}\right\|_{1}^{2}+\left\|q_{n} x_{n} q_{n}\right\|_{1}^{2} \\
& \geqslant\left\|p_{n} x_{n} p_{n}\right\|_{1}^{2}+\left(\left\|p_{n} x_{n} q_{n}\right\|_{1}+\left\|q_{n} x_{n} p_{n}\right\|_{1}+\left\|q_{n} x_{n} q_{n}\right\|_{1}\right) / 3 .
\end{aligned}
$$

So,

$$
\begin{aligned}
\left\|x_{n}-x\right\|_{1} & \leqslant\left\|p_{n} x_{n} p_{n}-x\right\|_{1}+\left\|p_{n} x_{n} q_{n}\right\|_{1}+\left\|q_{n} x_{n} p_{n}\right\|_{1}+\left\|q_{n} x_{n} q_{n}\right\|_{1} \\
& \leqslant\left\|p_{n} x_{n} p_{n}-x\right\|_{1}+3\left(\left\|x_{n}\right\|_{1}^{2}-\left\|p_{n} x_{n} p_{n}\right\|_{1}^{2}\right) .
\end{aligned}
$$

By (i) and (iv), the expression on the right-hand side of the last inequality tends to zero. This completes the proof of the theorem.

\section{REFERENCES}

1. J. Arazy, Basic sequences, embeddings and the uniqueness of the symmetric structure in unitary matrix spaces, J. Funct. Anal. (to appear).

2. , On the geometry of the unit ball of unitary matrix spaces, Integral Equations Operator Theory (to appear).

3. H. R. Grümm, Two theorems about $C_{p}$, Rep. Math. Phys. 4 (1973), 211-215.

4. N. Tomczak-Jaegermann, The moduli of smoothness and convexity and Rademacher averages of trace classes $S_{p}(1<p<\infty)$, Studia Math. 50 (1974), 163-182.

Department of Mathematics, University of Illinois at Urbana-Champaign, Urbana, Illinois 61801

Current address: Department of Mathematics, University of Haifa, Haifa, Israel 